\title{
Clustering and artificial neural networks: Classification of variable lengths of Helminth antigens in set of domains
}

\author{
Thiago de Souza Rodrigues ${ }^{1}$, Lucila Grossi Gonçalves Pacífico², Santuza Maria Ribeiro Teixeira², \\ Sérgio Costa Oliveira ${ }^{2}$ and Antônio de Pádua Braga ${ }^{1}$ \\ ${ }^{1}$ Universidade Federal de Minas Gerais, Departamento de Engenharia Eletrônica, \\ Minas Gerais, MG, Brazil. \\ ${ }^{2}$ Universidade Federal de Minas Gerais, Departamento de Bioquímica e Imunologia, \\ Minas Gerais, MG, Brazil.
}

\begin{abstract}
A new scheme for representing proteins of different lengths in number of amino acids that can be presented to a fixed number of inputs Artificial Neural Networks (ANNs) speel-out classification is described. K-Means's clustering of the new vectors with subsequent classification was then possible with the dimension reduction technique Principal Component Analysis applied previously. The new representation scheme was applied to a set of 112 antigens sequences from several parasitic helminths, selected in the National Center for Biotechnology Information and classified into fourth different groups. This bioinformatic tool permitted the establishment of a good correlation with domains that are already well characterized, regardless of the differences between the sequences that were confirmed by the PFAM database. Additionally, sequences were grouped according to their similarity, confirmed by hierarchical clustering using ClustalW.
\end{abstract}

Key words: bioinformatics, artificial neural networks, clustering, helminth antigen, domain.

Received: August 15, 2003; Accepted: August 20, 2004.

\section{Introduction}

Artificial Neural Networks (ANNs) and clustering techniques have been applied to genomic and proteomic studies in the last few years. One of the main difficulties in applying these techniques to protein sequence analysis is the variable length of the sequences and the high dimensionality. Since ANNs have a fixed number of inputs, classification of variable length sequences demands an appropriate representation in order to be processed.

Herein, we propose a new representation scheme for variable length high dimensional protein sequences to be applied to a set of pre-selected antigens. The principle of this new method is to create a fixed size image matrix $M$ for each protein sequence that represents the frequency of occurrence of all pairs of amino acids. Regardless of the original sequence size, the new representation scheme consists of generating a vector with 400 elements for each antigen. In the present study, only pairs of amino acids were consid-

Send correspondence to Antônio de Pádua Braga. Universidade Federal de Minas Gerais, Departamento de Engenharia Eletrônica, Caixa Postal 209, 30161-970 Pampulha, Belo Horizonte, MG, Brazil. E-mail: apbraga@cpdee.ufmg.br. ered, but the method can be applied to higher dimension partitions of the input data.

After obtaining an image vector for each antigen, they were all mapped onto a lower dimensional space using Principal Component Analysis (PCA) (Braga et al., 2000), (Haykin, 1999). The reduced dimension data resulted in lower computational costs and visualization in two and three dimensions, without relevant loss in the original distributions. In the experiment, the input dimension was reduced to $5(1.25 \%$ of the original dimension of matrix $M)$. Clustering techniques such as K-Means (Linkas et al., 2003), (Braga et al., 2000), and hierarchical clustering (Jain and Murty, 1999) were then applied to the reduced dimension vectors. The clusters obtained with the reduced data were consistent with those generated by hierarchical clustering of the original sequences and also with that generated by ClustalW (EBI). A qualitative analysis of the domains present in the elements of each cluster generated by $K$-Means was carried out taking into account the information available in the PFAM database (PFAM). Most of the sequences grouped into the same cluster have the same domain according to PFAM. 


\section{Material and Methods}

\section{Data analysis}

The data analysis and the new representation scheme were applied to a set of pre-selected sequences of 19 different antigens from various helminth parasites, obtained from National Center for Biotechnology Information (NCBI). Table 1 presents 19 helminths and the corresponding number of selected antigens of each one, resulting in $112 \mathrm{se}$ quences. The antigens chosen here were selected from the $N C B I$ database. These antigens are molecules targeted for new vaccine diagnostic reagents and drug discovery. The majority of these proteins have known functions which allow us to compare their classification using $K$-Means with PFAM.

The wide range of sequence sizes of the input data can be observed in Figure 1 that shows the number of amino acids that compose the polypeptide for each antigen. There are small sequences with sizes as short as 60 as well as others ranging from 400 to 800 amino acids. In order to treat the current classification and clustering problems with $A N N s$, it is necessary to define an appropriate coding scheme, which would make it possible to map the variable length of the data into the fixed size ANN inputs. The representation scheme presented here, was designed to overcome this difficulty.

Since the search for regularities in the input data could help in the new coding scheme, the data present in Figures 2 and 3 were generated. In Figure 2, the number of occurrences of each amino acid in all sequences is presented and in Figure 3, the amino acid concentration along the sequences are presented. It was observed that there is no prominent amino acid among all antigens sequences. It was observed that methionine is concentrated at the beginning of the sequences, as expected, and alanine, lysine and leucine appear in high concentrations, but well distributed along all antigens sequences. There were no new findings in the analysis of the input data that could help in the creation of a coding scheme to represent sequences in a proper manner to deal with $A N N s$. Thus, a general coding proce-

Table 1 - Helmiths and the number of sequences available for each one.

\begin{tabular}{lrlr}
\hline Helminth & $n$ Helminth & $n$ \\
\hline Taenia solium & 18 & Trichinella spiralis & 2 \\
Taenia ovis & 7 & Taenia crassiceps & 1 \\
Schistosoma japonicum & 13 & Fasciola hepatica & 4 \\
Schistosoma haematobium & 1 & Nippostrongylus brasiliensis & 4 \\
Echinococcus multilocularis & 13 & Clonorchis sinensis & 3 \\
Echinococcus granulosus & 22 & Ascaris suum & 2 \\
Trichostrongylus colubriformis & 2 & Toxocara canis & 1 \\
Paragonimus westermani & 1 & Onchocerca volvulus \\
Trichuris trichiura & 1 & Nippostrongylus brasiliensis & 5 \\
Wuchereria bancrofti & 1 & & 11 \\
\hline
\end{tabular}

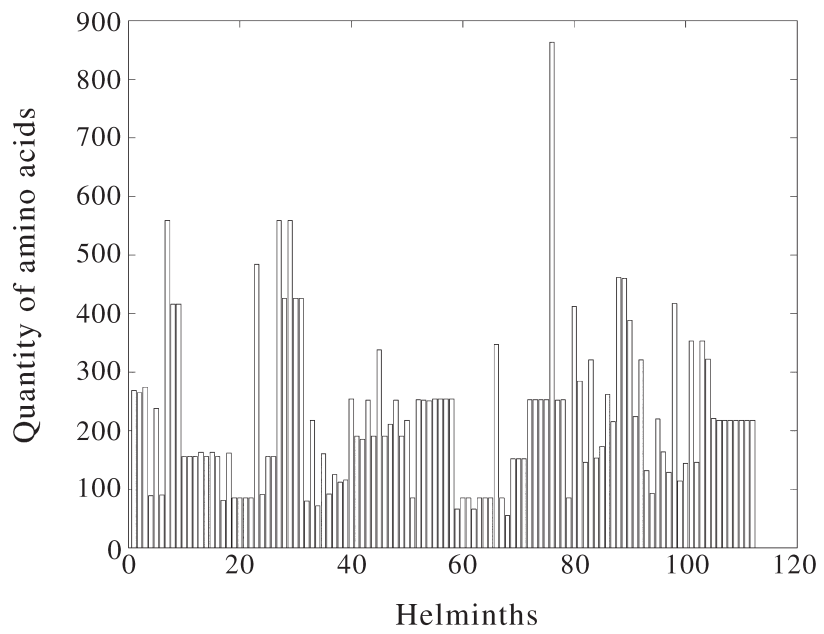

Figure 1 - Length of the analyzed antigens.

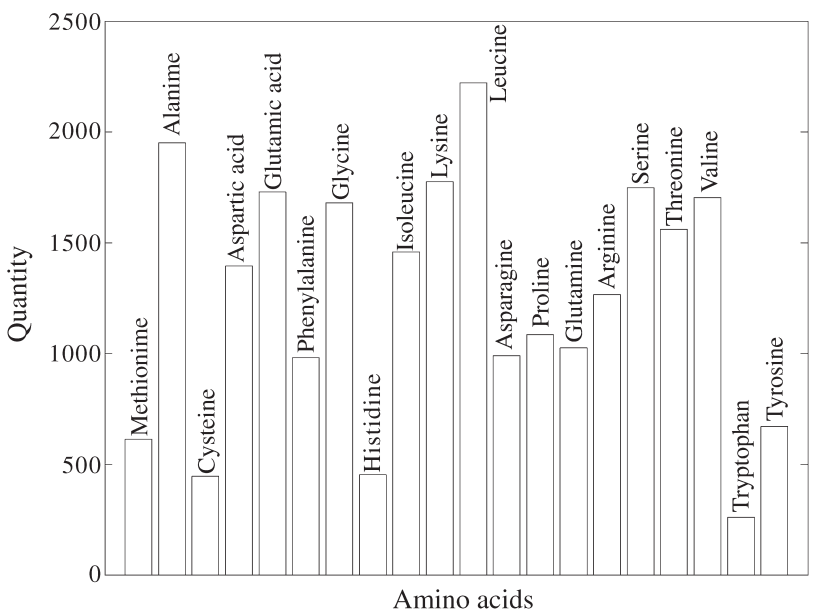

Figure 2 - Number of amino acids in the analyzed antigens.

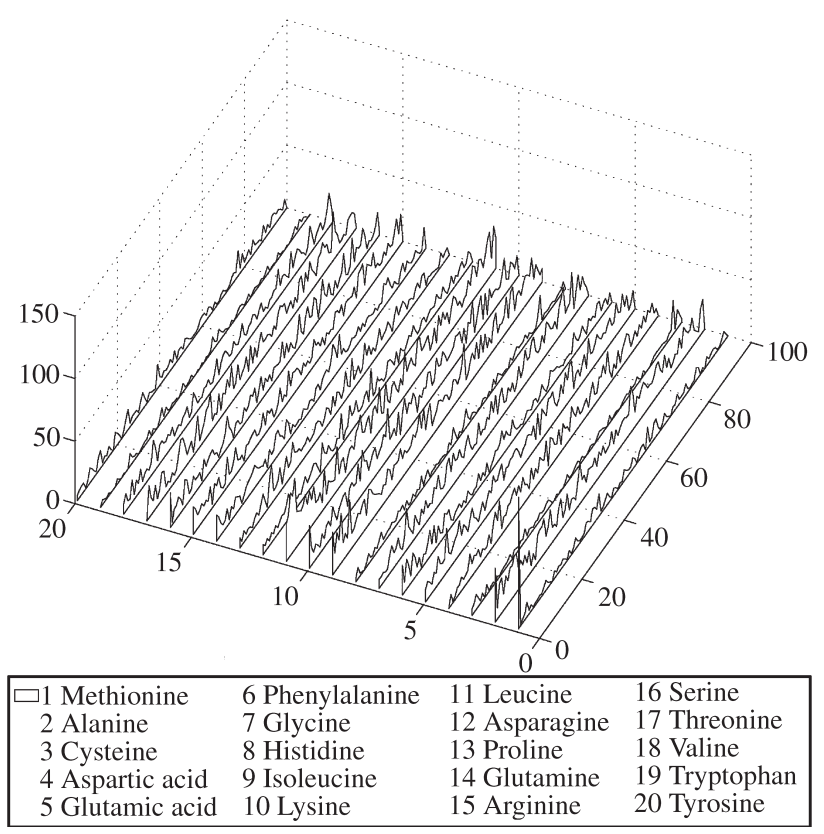

Figure 3 - Amino acid distribution throughout the antigens. 
dure was proposed, that can be applied to any sequence of amino acids, regardless of its origin, as shown in Material and Methods. As an extension of this new approach, Results and Discussion shows the application of $P C A$ and clustering by $K$-Means to the coded input data.

\section{Sequence coding by sliding window - SCSW}

In order to maintain the same length for all antigens, regardless of the number of amino acids, the coding scheme proposed consists on building an Image Matrix $M$ with 20 rows and 20 columns where each row and column are associated with a different amino acid. Each matrix element $\mathrm{M}_{\mathrm{ij}}$ contains the number of times the pair of amino acids $i$ and $j$ appear in the antigen being coded. In order to generate Image Matrix $M$, a sliding window that covers 2 amino acids at a time scans the sequence from the beginning to the end. For each pair of amino acids found, the corresponding matrix element is increased. The sliding window then shifts right one amino acid and counts the next pair, and so on.

Figure 4 and Table 2 show one of the antigens from the helminth Clonorchis sinensis (Yang et al., 2000) and the corresponding image matrix, respectively. It can be observed in Figure 4 that there are 24 occurrences of a pair of amino acids $A Q$. The correspondent position in matrix $M$ is represented by row 2 (amino acid $A$ ) and column 14 (amino acido $Q$ ), which contains the value 24 .

After all 112 antigens are coded, the 20 rows of each 20 by 20 matrix are transformed into a single vector with
MKFLKLVIIGALFLNVLCLDGGAQPPKSGDGGAQPPKSGDGGAQPPKSGDGGAQPPKS GDGGAQPPKSGDGGAQPPKSGDGGAQPPKSGDGGAQPPKSGDGGAQPPKSGDGGAQ PPKSGDGGAQPPKSGDGGAQPPKSGDGGAQPPKSGDGG AQPPKSGDGGAQPPKSGD GGAQPPKSGDGGAQPPKSGDGGAQPPKSGDGGAQPPKSGDGG AQPPKSGDGGAQPPK SGDGGAQPPKSGDGGAQPPKSGAQRPFSHWIAGWFLVPLEVKASDHF

Figure 4 - Clonorchis sinensis GRCSP antigen.

400-elements. Finally, all 400-elements vectors are aligned, generating a matrix with 112 rows, each one representing an antigen. Since all matrices are sparse, there is room for dimensionality reduction. As shown in the next section, the 112 by 400 matrix is reduced to a lower dimensional space by using Principal Component Analysis.

\section{Dimensionality reduction and clustering}

Due to the high dimensionality of input data, many problems may arise regarding computation time. Therefore, it is desirable to reduce the dimension to a manageable size, maintaining as much of the original information as possible. The sparseness of the image matrices indicates that dimensionality reduction would be possible without much loss of information. We have chosen Principal Componente Analysis (PCA) (Haykin, 1999), (Matthew and Calvo, 1998) to be applied in this study. Compared to other techniques for dimensionality reduction available in the literature, such as Factor Analysis (Fodor, 2002), Projection Pursuit (Fodor, 2002) and Vector Quantization (Gray, 1984), PCA has the advantage of conceptual simplicity, since it involves finding the best linear transforma-

Table 2 - Image matrix.

\begin{tabular}{|c|c|c|c|c|c|c|c|c|c|c|c|c|c|c|c|c|c|c|c|c|}
\hline & $\mathrm{M}$ & A & $\mathrm{C}$ & $\mathrm{D}$ & $\mathrm{E}$ & $\mathrm{F}$ & $\mathrm{G}$ & $\mathrm{H}$ & $\mathrm{I}$ & $\mathrm{K}$ & $\mathrm{L}$ & $\mathrm{N}$ & $\mathrm{P}$ & $\mathrm{Q}$ & $\mathrm{R}$ & $\mathrm{S}$ & $\mathrm{T}$ & V & W & $\mathrm{Y}$ \\
\hline $\mathrm{M}$ & 0 & 0 & 0 & 0 & 0 & 0 & 0 & 0 & 0 & 1 & 0 & 0 & 0 & 0 & 0 & 0 & 0 & 0 & 0 & 0 \\
\hline A & 0 & 0 & 0 & 0 & 0 & 0 & 0 & 0 & 0 & 0 & 1 & 0 & 0 & 24 & 0 & 1 & 0 & 0 & 0 & 0 \\
\hline $\mathrm{C}$ & 0 & 0 & 0 & 0 & 0 & 0 & 0 & 0 & 0 & 0 & 1 & 0 & 0 & 0 & 0 & 0 & 0 & 0 & 0 & 0 \\
\hline $\mathrm{D}$ & 0 & 0 & 0 & 0 & 0 & 0 & 23 & 1 & 0 & 0 & 0 & 0 & 0 & 0 & 0 & 0 & 0 & 0 & 0 & 0 \\
\hline $\mathrm{E}$ & 0 & 0 & 0 & 0 & 0 & 0 & 0 & 0 & 0 & 0 & 0 & 0 & 0 & 0 & 0 & 0 & 0 & 1 & 0 & 0 \\
\hline $\mathrm{F}$ & 0 & 0 & 0 & 0 & 0 & 0 & 0 & 0 & 0 & 0 & 3 & 0 & 0 & 0 & 0 & 1 & 0 & 0 & 0 & 0 \\
\hline $\mathrm{G}$ & 0 & 25 & 0 & 22 & 0 & 0 & 23 & 0 & 0 & 0 & 0 & 0 & 0 & 0 & 0 & 0 & 0 & 0 & 1 & 0 \\
\hline $\mathrm{H}$ & 0 & 0 & 0 & 0 & 0 & 1 & 0 & 0 & 0 & 0 & 0 & 0 & 0 & 0 & 0 & 0 & 0 & 0 & 1 & 0 \\
\hline I & 0 & 1 & 0 & 0 & 0 & 0 & 1 & 0 & 1 & 0 & 0 & 0 & 0 & 0 & 0 & 0 & 0 & 0 & 0 & 0 \\
\hline $\mathrm{K}$ & 0 & 1 & 0 & 0 & 0 & 1 & 0 & 0 & 0 & 0 & 1 & 0 & 0 & 0 & 0 & 23 & 0 & 0 & 0 & 0 \\
\hline $\mathrm{N}$ & 0 & 0 & 0 & 0 & 0 & 0 & 0 & 0 & 0 & 0 & 0 & 0 & 0 & 0 & 0 & 0 & 0 & 1 & 0 & 0 \\
\hline $\mathrm{P}$ & 0 & 0 & 0 & 0 & 0 & 1 & 0 & 0 & 0 & 23 & 1 & 0 & 23 & 0 & 0 & 0 & 0 & 0 & 0 & 0 \\
\hline Q & 0 & 0 & 0 & 0 & 0 & 0 & 0 & 0 & 0 & 0 & 0 & 0 & 23 & 0 & 1 & 0 & 0 & 0 & 0 & 0 \\
\hline $\mathrm{R}$ & 0 & 0 & 0 & 0 & 0 & 0 & 0 & 0 & 0 & 0 & 0 & 0 & 1 & 0 & 0 & 0 & 0 & 0 & 0 & 0 \\
\hline $\mathrm{S}$ & 0 & 0 & 0 & 1 & 0 & 0 & 23 & 1 & 0 & 0 & 0 & 0 & 0 & 0 & 0 & 0 & 0 & 0 & 0 & 0 \\
\hline $\mathrm{T}$ & 0 & 0 & 0 & 0 & 0 & 0 & 0 & 0 & 0 & 0 & 0 & 0 & 0 & 0 & 0 & 0 & 0 & 0 & 0 & 0 \\
\hline $\mathrm{V}$ & 0 & 0 & 0 & 0 & 0 & 0 & 0 & 0 & 1 & 1 & 1 & 0 & 1 & 0 & 0 & 0 & 0 & 0 & 0 & 0 \\
\hline W & 0 & 0 & 0 & 0 & 0 & 1 & 0 & 0 & 1 & 0 & 0 & 0 & 0 & 0 & 0 & 0 & 0 & 0 & 0 & 0 \\
\hline $\mathrm{Y}$ & 0 & 0 & 0 & 0 & 0 & 0 & 0 & 0 & 0 & 0 & 0 & 0 & 0 & 0 & 0 & 0 & 0 & 0 & 0 & 0 \\
\hline
\end{tabular}


tion in the mean-square error sense (Fodor, 2002). In order to obtain this transformation and axes elimination, the method calculates first the cross-correlation matrix of the input data, which is then decomposed into a product of matrices that contains the singular values (Press, 1988), which are sorted according to their relevance. The most relevant axes associated with the larger singular values are maintained, and the least relevant ones associated with the smaller singular values are discarded. The new coordinates system is formed only by the most relevant axes that result in dimension reduction.

Since the singular values have a smooth variation, as observed in Figure 5, there is a trade-off between the number of dimensions included and the resulting loss of information. The larger the variance (singular value) of the projection into a given axis, the more information that axis contains. Axes with null values of variance contain no relevant information about the input distribution of data. As indicated by the data of Figure 5, there are non-null variances until dimension 73 , which indicate that the 400 -elements vector can be transformed into 73 -elements vectors without loss of information. In order to obtain reduction to a smaller dimension there will be some loss of information, but the cut-off point is a user-defined parameter. In the tests carried out for the antigens, for dimensions larger or equal to 5 , there was no change in the membership of clustered data. So, with the application of $P C A$, it was possible to reduce the dimension of the input data from 400 to $5(1.25 \%$ of the original dimension of the image matrix $M$ ).

After transforming the 112 by 400 into new 112 by 5 matrices the data was then clustered into 40 different groups of antigens. The method used for grouping the antigens was $K$-Means algorithm, (Linkas et al., 2003), (Braga et al., 2000) which consists of finding $K$ sets of data with minimum variance. The parameter $K$ can be set a priori by the user, although there are variations of the original algorithm that find the value of $K$ adaptively (Jain, Murty and

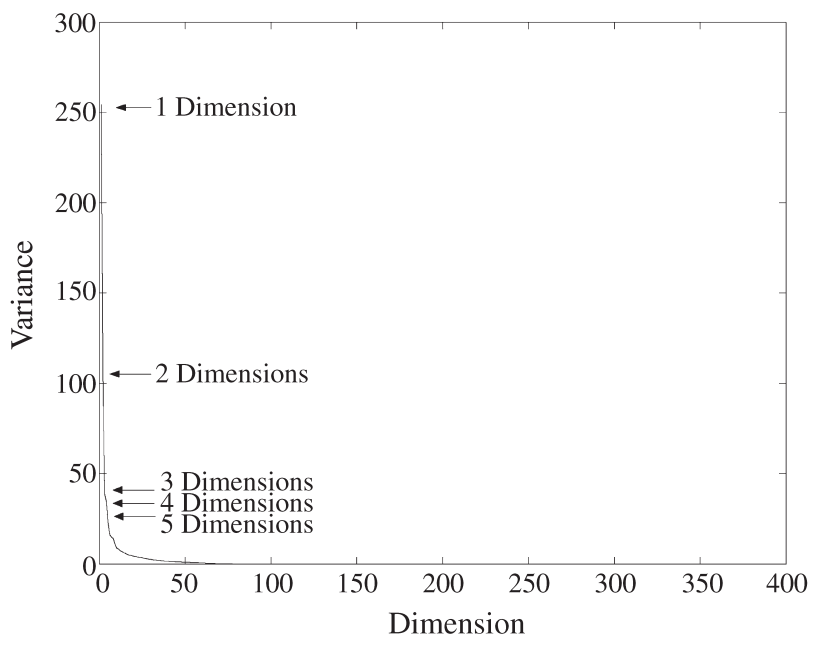

Figure 5 - Singular values (variances) of the decomposed crosscorrelation matrix.
Flynn 1999). For clustering, the value of $K$ was set closer to the number of domains within the 112 antigens.

Both methods, Principal Component Analysis and $K$-Means, were implemented in MATLAB (Mathworks) due to its simplicity for prototyping and the availability of many libraries of mathematical functions necessary to implement the algorithms.

\section{Results and Discussion}

Data analysis was performed and each gene was clustered according to its domain. This result was compared and confirmed using the PFAM database. The fifteen clusters found are summarized in Table 3.

The RRM domain (found in two antigens) is characteristic of a variety of RNA binding proteins, including heterogeneous nuclear ribonucleoproteins, proteins implicated in regulation of alternative splicing, and components of small nuclear ribonucleoproteins (Brandziulis et al., 1989) (accession number in PFAM: PF00076). The FERM domain (present in three antigens) is found in a number of cytoskeletal-associated proteins that associate with various polypeptides at the interface between the plasma membrane and the cytoskeleton. It is a conserved $\mathrm{N}$-terminal domain of about 150 residues, involved in the linkage of cytoplasmic proteins to the membrane (Rees et al., 1990), (Funayama et al., 1991) (accession number in PFAM: PF00373). The SCP domain (found in three antigens) belongs to proteins involved in sperm maturation and glioma pathogenesis-related processes (Mizuki and Kasahara, 1992) (accession number in: PF00188).

The fourth domain analyzed is the EF hand domain (found in eight antigen) consisting of a twelve residue loop flanked on both sides by a twelve residue-helical domain. It is known to be a calcium binding domain and may consti-

Table 3 - Domains and corresponding antigens.

\begin{tabular}{lc}
\hline Domains & Number of polypeptides \\
\hline RRM & 2 \\
FERM & 3 \\
SCP & 3 \\
EF Hand & 8 \\
SH3 & 5 \\
Four TRANSMEMBRANE & 9 \\
Fibronectin Type III & 9 \\
Extensin & 1 \\
Annexin & 2 \\
Myosin & 1 \\
ShTK & 3 \\
Calreticulin & 1 \\
TIM & 2 \\
Taeniidae & 18 \\
No Match & 5 \\
\hline
\end{tabular}


tute a cross-reaction with IgE (Dunne et al., 1998) (accession number in PFAM: PF00036). The Src-homology 3 domain (SH3) (found in five antigens) is a small protein domain of about 60 amino acid residues, probably folded into $b$ - sheets. SH3 domain is present in a large number of eukaryotic proteins that are involved in signal transduction, cell polarization, protein-protein and membranecytoskeleton interactions (Musacchio et al., 1992) (accession number in PFAM: PF00018).

A domain found in nine antigens is the Four TRANSMEMBRANE domain, which contains an $\mathrm{N}$ terminal transmembrane domain and three additional transmembrane regions. These sequences contain a number of conserved cysteine residues (Levy et al., 1991) (accession number in PFAM: PF00335). The Fibronectin type III domain (found in nine antigens) are also present in multi-domain glycoproteins found in a soluble form in plasma, and in an insoluble form in loose connective tissue and basement membranes. The fibronectins are involved in a number of important functions: e.g., wound healing; cell adhesion; blood coagulation; cell differentiation and migration; maintenance of the cellular cytoskeleton; and tumor metastasis (Skorstengaar et al., 1986) (accession number in PFAM: PF00041).

The Extensin domain (found in one antigen) also present in hydroxyproline-rich glycoproteins found in the plant extracellular matrix (accession number in PFAM: PF04554). The Annexin domain (found in two antigens) seems to be involved in cytoskeletal interactions, phospholipase inhibition, intracellular signaling, anticoagulation, and membrane fusion (Barton et al., 1991) (accession number in PFAM: PF00191). The Myosin domain (found in one antigen) consists of the coiled-coil myo$\sin$ heavy chain tail region. The coiled-coil is composed of the tail from two molecules of myosin, providing the structural backbone of the thick filament (Strehler et al., 1968) (accession number in PFAM: PF01576). The ShTK domain (found in three antigens) is also present in several $C$. elegans proteins, rich in cysteine residues, which probably form three disulphine bridges (accession number in PFAM: PF01549). The Calreticulin domain (found in one protein), characterizes this high-capacity calcium-binding protein, present in most tissues and located at the periphery of the endoplasmic and the sarcoplamic reticulum membranes (Michalak et al., 1992) (accession number in PFAM: PF00262). The Triosephosphate isomerase domain (TIM) (found in two antigens) is present in enzymes that play an important role in several metabolic pathways and is essential for efficient energy production (Knowles, 1991) (accession number in PFAM: PF00121).

The last two clusters are especially interesting. One contains twenty one antigens, eighteen of them belonging to the Taeniidae domain family and three with no matching result, these three polypeptides are likely to be part of the same the Taeniidae domain family. Another cluster containing five antigens, with no matching results, may provide an indication that these five polypeptides belong to the same family and are, thus, functionally related.
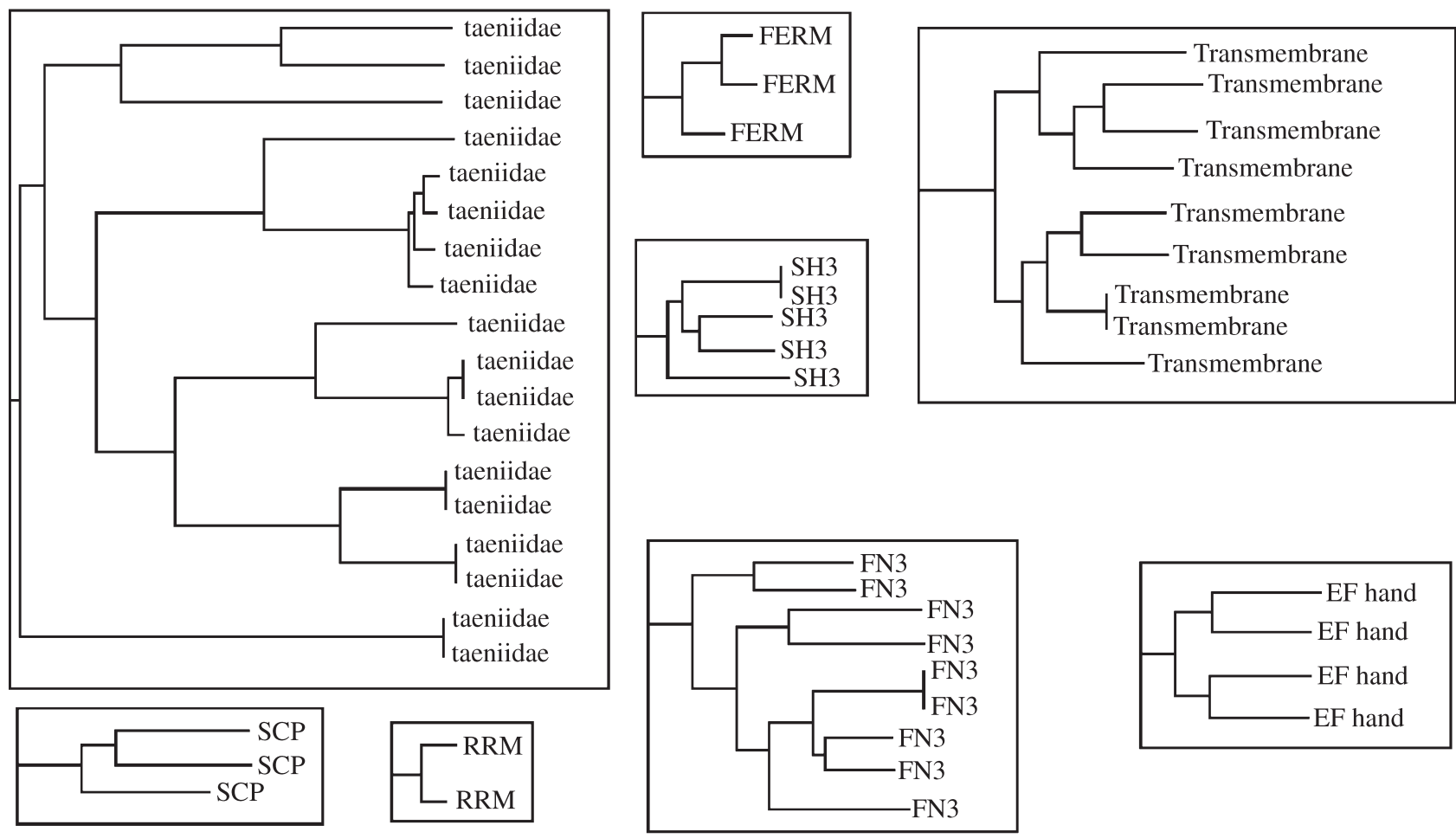

Figure 6 - Hierarquical clustering by ClustalW. 
The 37 antigens that are not referenced in Table 3 were not considered because the antigens were characterized by $P F A M$ as putative domain.

Using ClustalW for multiple alignment we verified that the antigens were grouped in the same clusters. Figure 6 shows parts of the hierarchical cluster displayed by ClustalW. Note that the clustering carried out by K-Means onto the SCSW scheme coincide with the clustering of ClustalW onto the original amino acids sequence, even with differences between the sequences. This shows that the codification scheme proposed in this study (SCSW), even though it is based in pairs of amino acids, retains enough information to enable us to correctly classify all the sequences.

This bioinformatics tool permitted us to establish a good correlation with domains that are already well characterized, regardless of the differences between the sequences. Additionally, our method is able to group the polypeptides in accordance with their similarity, using the new codification scheme SCSW. Our results show that the codification proposed can be useful in several applications involving Artificial Neural Networks, where the same data dimensionality is essential.

\section{References}

Bandziulis RJ, Swanson MS and Dreyfuss G (1989) RNA-binding proteins as developmental regulators. Genes \& Development 3:431-437.

Barton GJ, Newman RH, Freemont PS and Crumpton MJ (1991) Amino acid sequence analysis of the annexin super-gene family of proteins. Eur J Biochem 198:749-760.

Braga AP, Carvalho AF and Ludermir TB (2000) Redes Neurais Artificiais: Teoria e Aplicações. Livros Técnicos e Científicos, São Paulo, 262 pp.

Dunne DW and Hafalla JCR (1998) Identification of the Schistossoma japonicum 22.6-kDa antigen as a major target of the human $\operatorname{IgE}$ response: Similarity of IgE-binding epitopes to allergen peptides. Int Arch Allergy Immunol 117:94-104.

European Bioinformatics Institute (EBI), http://www.ebi.ac.uk/ clustalw.

Fodor IK (2002) A survey of dimensional reduction techniques. LLNL technical report $27 \mathrm{pp}$.
Funayama N, Nagafuchi A, Sato N and Tsukita S (1991) Radixin is a novel member of the band 4.1 family. The Journal of Cell Biology 115:1039-1048.

Gray RM (1984) Vector Quantization. IEEE ASSP MAGAZINE, Stanford, pp 4-29.

Haykin S (1999) Neural Networks: A comprehensive foundation. Prentice Hall, 900 pp.

Jain AK, Murty MN and Flynn PJ (1999) Data clustering: A review. ACM Computing Surveys 31:264-323.

Knowles JR (1991) Enzyme catalysis: Not different, just better. Nature 350:121-124.

Levy S, Nguyen VQ, Andria ML and Takahashi S (1991) Structure and membrane topology of TAPA-1. The Journal of Biological Chemistry 266:14597-14602.

Likas A, Vlassis N and Verbeek JJ (2003) The global $k$-means clustering algorithm. Pattern Recognition 36:451-461.

Mathworks, http://www.mathworks.com.

Matthe WR and Calvo RA (1988) Fast dimensionality reduction and simple PCA. Intelligent Data Analysis 2:203-214.

Michalak M, Milner RE, Burns K and Opas M (1992) Calreticulin. Biochemistry J 285:681-692.

Mizuki N and Kasahara M (1992) Mouse submandibular glands express an androgen-regulated transcript encoding an acidic epididymal glycoprotein-like molecule. Molecular and cellular endocrinology 98:25-32.

Musacchio A, Gibson T, Lehto VP and Saraste M (1992) SH3 An abundant protein domain in search of a function. FEBS Letters 307:55-61.

National Center for Biotechnology Information (NCBI),http://www.ncbi.nlm.nih.gov/pubmed.

Press W (1988) Numerical Recipes in C: The Art of Scientific Computing. Cambridge University Press 994 pp.

Protein Family database of alignments and HMMs (PFAM), http://www.sanger.ac.uk/Software/Pfam/.

Rees DJ, Ades SE, Singer SJ and Hynes RO (1990) Sequence and domain structure of talin. Nature 347:685-689.

Skorstengaard K, Jensen MS, Sahl P, Petersen TE and Magnusson S (1986) Complete primary structure of bovine plasma fibronectin. European Journal of Biochemistry 161:441-453.

Strehler EE, Strehler-Page MA, Perriard JC, Periasamy M and Nadal-Ginard B (1986) Complete nucleotide and encoded amino acid sequence of a mammalian myosin heavy chain gene. Evidence against intron-dependent evolution of the rod. Journal of Molecular Biology 190:291-317.

Yang HJ, Park SJ, Im KI and Yong TS (2000) Identification of a Clonorchis sinensis gene encoding a vitellaria antigenic protein containing repetitive sequences. Molecular Biochemistry Parasitology 111:213-216. 